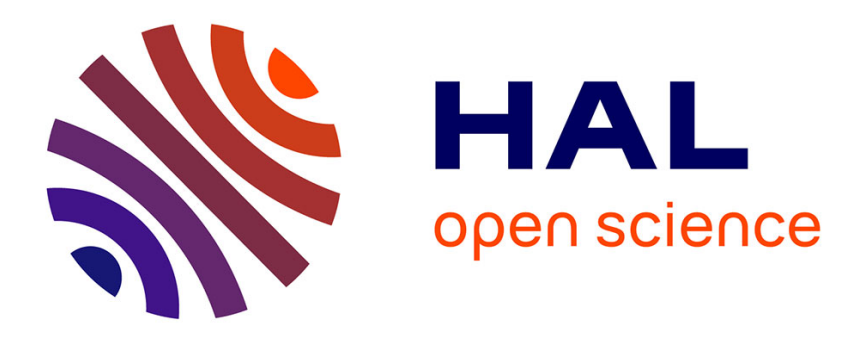

\title{
Fractal and Multifractal Characteristics of Propagating Cracks
}

\author{
V. Silberschmidt
}

\section{To cite this version:}

V. Silberschmidt. Fractal and Multifractal Characteristics of Propagating Cracks. Journal de Physique IV Proceedings, 1996, 06 (C6), pp.C6-287-C6-294. 10.1051/jp4:1996628 · jpa-00254456

\section{HAL Id: jpa-00254456 https://hal.science/jpa-00254456}

Submitted on 1 Jan 1996

HAL is a multi-disciplinary open access archive for the deposit and dissemination of scientific research documents, whether they are published or not. The documents may come from teaching and research institutions in France or abroad, or from public or private research centers.
L'archive ouverte pluridisciplinaire HAL, est destinée au dépôt et à la diffusion de documents scientifiques de niveau recherche, publiés ou non, émanant des établissements d'enseignement et de recherche français ou étrangers, des laboratoires publics ou privés. 


\title{
Fractal and Multifractal Characteristics of Propagating Cracks
}

\author{
V. Silberschmidt \\ Wilhelmstrasse 88, 38100 Braunschweig, Germany
}

\begin{abstract}
Specific features of crack propagation in random brittle materials are studied by means of lattice modelling. Emphasis of the investigation is on the analysis of the effect of spatial stochasticity in material properties' distribution on fracture characteristics. Local processes - evolution of ensembles of microdefects and its interaction with a crack- are introduced in terms of damage parameter and relevant evolution law. The influence of these processes on crack propagation is described as a crack-damage interaction: the criterion of local failure, causing the consequent crack growth, is introduced in the form of a critical damage value. Crack front morphology and time parameters of failure development are studied using the concepts of fractals and multifractals for three different cases of material's stochasticity (spatial randomness in properties' distribution). Characteristic features of energy release under crack growth are pointed out.
\end{abstract}

\section{INTRODUCTION}

A growth of a crack in real (quasi)brittle solids depends not only on their macroscopic non-uniformity (type of anisotropy, texture, etc.), but also on the development of the local - micro- and mesoscopical processes near its tip. The latter, in their turn, are sufficiently influenced by the crack-induced stress concentration. Such a self-action determines an important feature of the fracture - its self-similarity (scaling). This fact - alongside with the great interest to (and a large progress in) examination of the processes in random media in last two decades - stipulated the utilization of methods of statistical physics in fracture analysis (see reviews in [1] and respective references there). Still, the main part of such approaches, though providing very interesting results, is based on rather simplified media representations - for instance, pure elastic strings/beams - and failure conditions. Their another - and may be more important - disadvantage is the absence of mechanisms, describing stress concentration near a crack tip, though this was thoroughly studied by fracture mechanics and became a classical point in failure analysis $[2,3]$.

On the other hand, these statistical models supply effective tools for analysis of both local effects and random processes, that is very important for a study of crack propagation in real brittle materials. Parallel with these approaches the models of crack-microdefects interactions were intensively elaborated both in fracture mechanics and continuum damage mechanics (CDM) (see very informative review by Kachanov [4] and references there). But traditionally, such models deal with either a few defects with given positions/geometry, or with regular arrays of imperfections. One more way is the description of random media in terms of effective characteristics, exploiting such techniques as homogenization, etc. [5]. Thus, in all mentioned groups of approaches the randomness is reduced either to quasi-isotropy (with 
effective properties) or to a deterministic situation (may be only with more complicated distribution of defects/properties). Still, the simplest experiments on fracture of brittle specimens demonstrate, that the exact pictures of such a failure possess neither smoothness nor regularity. So, the analysis of random fracture "as it is" is of a sufficient interest. In this paper the stochastic crack propagation, is analysed using ideas of statistic physics, on one hand, and of CDM and fracture mechanics, on the other.

\section{MODEL}

Effect of local processes is especially vivid in failure analysis. The fractographic study of fractures demonstrate sufficient difference of fracture surfaces not only for materials with different type of deformational behaviour, but also for the groups of materials with resembling properties or even for the specimens of the same material.

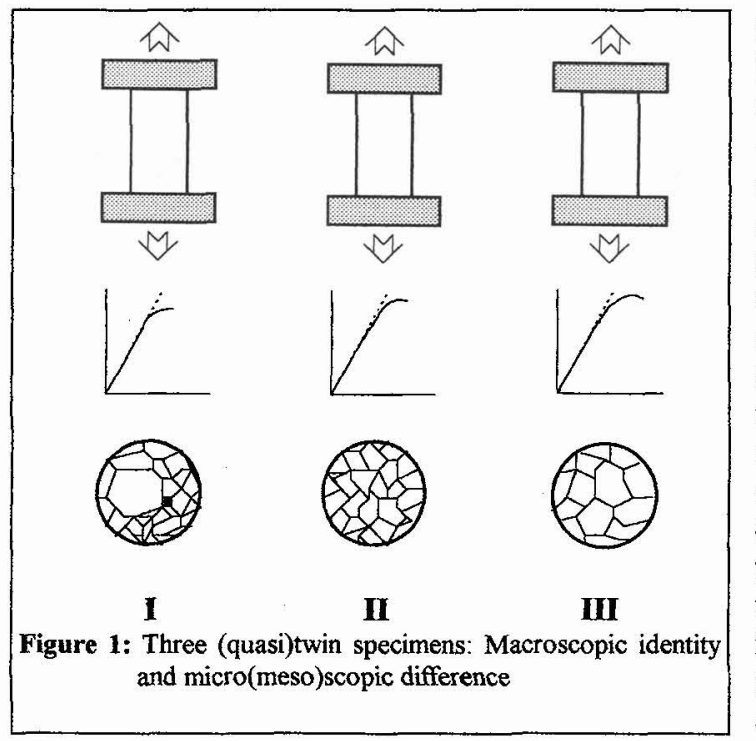

Figure 1 schematically presents the difference between two approaches to failure analysis. Let consider a simple tension test - up to total rupture - performed for three macroscopically identical specimens of the same material under equal external conditions. Results of this (macroscopic) treatment would probably demonstrate a closeness of elasticity module and more vivid scatter in the rupture parameter(s) (strain and/or stress to failure) - the middle row in Fig. 1. Still, the structural study (a lower row) can show the micro(meso)scopical difference of these specimens: the extent of structural randomness diminishes from the sample I to the sample III, the former having rather stochastic structure, while the latter - nearly isotropic. The stochasticity of material's properties on micro(meso)scale can be linked, for instance, with a non-uniform distribution of second phase, polydispersion of grain sizes, technological defects in composites, etc. A description of such types of structure in terms of micromechanics not necessarily leads to desirable estimation of fracture characteristics: transitions over a set of scale levels is a complicated problem. On the other hand, transition to averaged (effective) macroproperties, correctly estimating general behaviour of material, can result in an inadequate prediction of the crack morphology and of peculiarities in crack evolution. But there is also another way to introduce stochasticity direct into a model.

\subsection{Account for stochasticity}

Let consider that each specimen of Figure 1 can be characterized by an exact type of spatial distribution of its properties. Relevant distributions can be obtained by treatment of a set of specimens or of sections of the same specimen. In this paper a scatter in stiffness would be considered as a main factor, influencing -directly and indirectly - the non-uniform crack growth. Then the character of distribution function of stiffness - its half-width, for instance - can serve as a measure of stochasticity of material's properties. Discretization of a rectangular cross-section of a specimen under uniform tension (Fig. 1) into elements with dimensions, which satisfy the requirements of a representative volume; and distribution of stiffness randomly over them introduce an initial spatial randomness of the specimen. Three model cases of stochasticity with respective analytical presentation for relevant distribution functions are introduced 
in [6-8]. Let note, that the total stiffness of the cross-section - equal to a sum of stiffnesses of building it elements - for all three cases is the same, thus these specimens are "macroscopically identical".

An increase in the coefficient of distribution's half-width $K_{0.5}$ means respective decrease of the randomness of material ( $K_{0.5}=0.5$ being a case of total isotropy). So, let characterize each specimen of Fig. 1 by such a coefficient: 1) material with a high level of stochasticity in properties distribution specimen I - has $K_{0.5}=0.1683 ; 2$ ) for nearly isotropic material - specimen III - $K_{0.5}=0.4997 ; 3$ ) for an intermediate case $K_{0.25}=0.25$ (specimen II). Spatial randomness in material characteristics results in a non-uniform distribution of stress even under a homogenous external loading: with a part of load carried by an element being proportional to its stiffness in approximation of a plane strain the initial level of tensile stress in elements can be presented in a following form:

$$
\sigma_{m}^{1, j}=K_{m}^{i, j} \sigma_{\infty}
$$

where a pair of indexes $i \in\left[1, N_{1}\right]$ and $j \in\left[1, N_{2}\right]$ denotes a rectangular element of the $i$-th row and $j$ th column in the discretization of the cross-section; $K_{i n}^{i, j}=G^{i, j} /\left\langle G^{i, j}\right\rangle ; \sigma_{\infty}=$ const is the tensile stress acting far from the cross section under consideration. Here $G^{i, j}$ is a stiffness of element $(i, j) ;\langle\rangle$ means averaging over all $M=N_{1} \times N_{2}$ elements.

\subsection{Crack-damage interaction}

The scheme of accounting for interaction of processes of crack propagation and evolution of ensembles of micro(meso)scopical defects was described in details elsewhere [8-10], so its main aspects are only mentioned here:

1) Damage parameter and accumulation law. The problem's geometry (Fig. 1) make the use of scalar damage parameter $p^{i, j}$ possible. Though various approximations for kinetic equation can be applied, in this paper the quasi-linear form of it- with a self-action - is utilized:

$$
\frac{d p^{i, j}}{d t}=A \sigma^{i, j}+B p^{i, j}
$$

Here the rate of growth of damage - calculated for each element - is proportional not only to the stress, but also to an achieved local defectness level itself.

2) Local failure criterion. The overcoming of a critical value by a damage level for a given element results in its faihure. This causes the decrease of element's stiffness (bearing capacity): $G^{i, j} \rightarrow \varepsilon G^{i, j}$, $\varepsilon<1$ (in calculations below $\varepsilon=0$ ).

3) Concepts of stress-concentration coefficients and of local stress-intensity factors (SIF). Both the stress concentration near a tip of propagating crack and stress redistribution processes, linked with the local failure of elements, are accounted for in terms of stress-concentration coefficients:

$$
\sigma^{i, f}=K^{i, j} \sigma_{t m}^{i, j}
$$

The non-uniformity of material properties' spatial distribution (and resulted randomness in both stress levels and, consequently (see Eq. (2)), local dynamic of damage accumulation) causes the difference in crack length along its front - in contrast to traditional crack models in fracture mechanics, where cracks fronts are smooth. So, local SIFs are introduced by integrating of a respective relation for a mode-I crack $[2,3] \sigma_{z z}=K_{1} / \sqrt{2 \pi y}$ over elements in rows (initial V-notch occupies $n_{i w}$ columns of elements; in our case an axis $z$ coincides with a tension direction, $y$ is parallel to rows). Then 


$$
K^{i, J}=\frac{\bar{G}^{i}}{\widetilde{G}^{\prime}} K_{\mathrm{I}}^{i} \sqrt{\pi d_{y}}\left(\sqrt{j+1-n^{i}}-\sqrt{j-n^{i}}\right), j \geq n^{i}
$$

Here $\bar{G}^{i}=\sum_{i=n_{i b}+1}^{N_{i}} G^{i, j}$ is initial stiffness of the $i$-th row, while $\widetilde{G}^{i}$ being its current value; $K_{\mathrm{I}}^{i}$ can be approximated by known relations for a case under study from handbooks on SIF [11]; $l_{y}$ is an elements' length in $y$ direction; $n^{i}$ is a number of elements, occupied by crack, in the $i$-th row.

Thus, crack propagation and stress redistribution lead to increasing of stress values in elements, accelerating, in its turn, damage growth there and consequent failure of these elements in front of a crack. In a case of a totally isotropic material this trend would result in a shift of crack front as a straight line parallel to its initial position (as is foreseen by the classical fracture mechanics) $[2,3]$. But the nonuniform realization of local processes near a crack tip and their complicated interaction result in its nontrivial morphology (well-known not only from respective experiments, but also from the everyday experience). This presupposes both the modification of traditional characteristics of 'fracture and introduction of new ones.

\section{RESULTS}

\subsection{Characteristics of cracks}

Numerical calculations, based on the above model, approved a sufficient difference in crack's characteristics for three specimens under study. The level of indentness of a crack front increases with the growth of the randomness in properties' distributions $[8,12]$; let note, that a typical for fracture mechanics case of the propagation of front as a line parallel to the tip of initial V-notch under uniaxial tension is also obtained within the discussed approach - as a limiting case of material's uniformity with $K_{0.3}=0.5$. The principal feature of a proposed approach (in contrast to traditional ones) is the account for damage accumulation - in form of Eq. (2) - and an introduction of a local failure criterion, that naturally includes a time axis into model consideration.

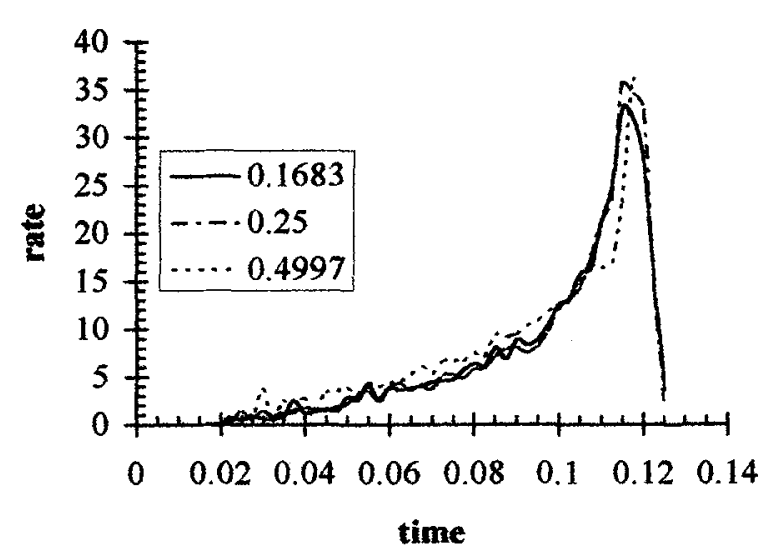

Figure 2: Change of mean crack rate (for various values of $K_{0.5}$ ) with time
Thus, this description allows also to analyse temporal properties of crack propagation (here the slow crack growth - not a dynamic fracture - is modelled). Introducing a mean crack length as $\bar{l}=l_{y}\left\langle n^{i}\right\rangle$ (another characteristics of crack front position and their change with time were studied in $[8,12]$ ), the evolution of a mean crack rate for three types of materials stochasticity - specimens from Fig. 1 - can be obtained (Figure 2). Here and below all results are calculated on the net of 10000 elements. An increase in material's uniformity is reflected by the higher level of fluctuations in a mean crack rate. This coincides with our previous results, that demonstrated the appearance of a jump-like character 
of crack propagation for a case of lower material randomness [8]. A disorder smoothes out these fluctuations; local jump-like acts of crack growth are desynchronized in this case.

\subsection{Sealing characteristics of crack propagation process}

An irregularity of crack fronts in stochastic brittle media presupposes an utilization of adequate tools for their characterization. It was shown $[6,8-10,12]$, that morphology of fronts is of self-similar (more precisely - self-affine) character and as thus has the fractal character. The fractal dimension $D_{f}$ is linked with the stochasticity type of material: for a $2 d$-problem under study it changes from 2 to 1 under increase in material homogeneity with a natural limiting case $D_{f} \rightarrow 1$ for a total isotropy. The latter is obvious: in such a case crack propagates with a front being a straight line, which has a trivial fractal dimension equal unity. Thus, there exists a direct linkage between the extent of material's stochasticity and scaling characteristic of crack geometry: various statistical realization for the same type of stiffness distribution function have very close values of fractal dimension.

Another kind of spatial scaling demonstrates the character of load (stress) distribution near such cracks. Such distributions are characterized by an infinite number of scaling parameters for their moments and are thus multifractal. The shape of multifractal spectrum $f(\alpha)$ is determined in such a case by randomness in properties of media [13]: the width of these spectra declines with the growth of material's uniformity.

Stochastic crack propagation possesses also a temporal scaling. An estimation of the time-tofracture of specimens - the moment of the exit of crack front on the side, opposite to an initial notch - has shown [14], that though the scatter of this parameter for a changing stochasticity extent reaches tens per cent, the scaling exponent in relation 'time-to-fracture $\nu s$. stress' in a wide range of $\sigma_{\infty}$ is the same and very close to minus unity. Thus, in double logarithmic coordinates respective graphs are straight lines parallel each other.

\subsection{Dynamics and scaling of energy release}

The next step in study of stochastic crack propagation is the analysis of elastic energy release under such fracture process. The data on the stress level for each element in a cross-section for any time step with the local failure criterion allows to get a quantitative estimation for amount of energy, being released in a respective moment of time. Results of numerical simulation for three specimens under study are presented in Figure 3.

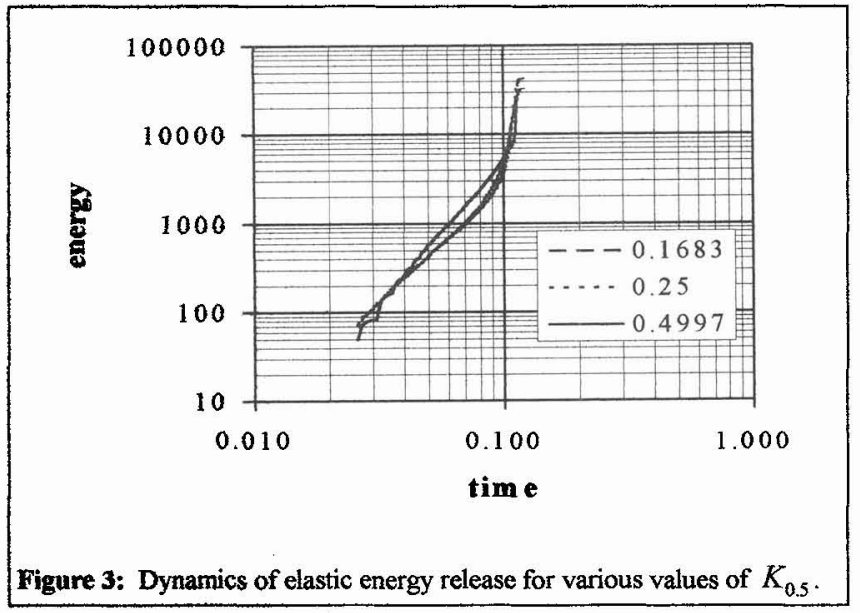

It is obvious, that there are two scaling regimes in energy release; a) initial stage of crack propagation and b) final, prerupture, stage. The first stage is characterized by the value of scaling exponent, that is nearly an order of magnitude less than one for a final stage. The quasi-isotropic material (specimen III, Fig. 1) has a greater level of scaling exponent, while two other specimens have very close values: a better regulated (synchronized) character of local failure of elements results in accelerated energy release.

For a more precise treatment of data on energy release under stochastic 
crack propagation a multifractal analysis is performed with a use of so-called canonical method (see, for instance, [15-18] and references there). According to it, the $f-\alpha$ relation - a singularity spectrum of $q$ moments of distribution - is obtained as parametrically depending on $q$ :

$$
\begin{gathered}
\alpha(q)=\frac{\sum_{i=1}^{N} p_{i} \log \mu_{i}}{\log \varepsilon}, \\
f(\alpha(q))=\frac{\sum_{i=1}^{N} \mu_{i} \log \mu_{i}}{\log \varepsilon},
\end{gathered}
$$

where $p_{i}=E_{i} / E_{\text {tot }} ; \mu_{i}=p_{i} / \sum_{i=1}^{N} p_{i}^{q} ; \varepsilon=1 / N$. Here $E$ is the amount of elastic energy, being released on the $i$-th time step $(i \in[1, N], N$ being the number of steps, covering a time interval from the first event of a local (element's) failure till the total rupture of a specimen), $E_{s t}$ is the total energy, released under the whole process of crack propagation.

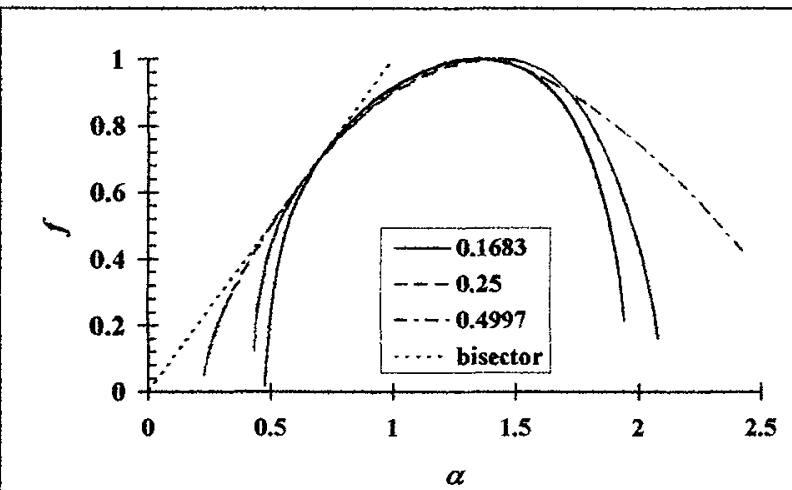

Figure 4: Multifractal spectra of energy release under crack propagation in three various stochastic media (various values of $K_{0.5}$ ).
Figure 4 presents results of application of a canonical method to data on energy for three various cases of stochasticity. Obtained functions $f-\alpha$ possess all the properties of multifractal spectra: they are cup convexes, laying under bisector and having with it only one point of connection. But here - in contrast to spatial multifractal distributions - an increase in material uniformity is related to growth of the spectrum's width as a sequence of syncronization of elementary acts of energy release for separate elements. Thus, disorder causes more isotropic temporal processes.

\subsection{In-plane roughness of crack front}

Additional information about crack morphology - its extent of indentness - can be obtained by analysis of in-plane roughness of its front. Respective procedure was proposed in [19] based on the general scheme for characterization of self-affine profiles [20]. Modifying it for a case under study, the following equation for obtaining of a roughness exponent can be introduced:

$$
z_{\max }(r)=\left.l_{y}\left\langle\left.\max n^{i}\left(r^{\prime}\right)\right|_{x<r^{\prime}<x+r}-\left.\min n^{i}\left(r^{\prime}\right)\right|_{x<r^{\prime}<x+r}\right\rangle\right|_{x} .
$$

Here $x$ is a co-ordinate along the crack front and $r$ is a window size. Thus, a parameter $z_{\max }(r)$ is a projection to the $y$-axis of a distance between the furthest - with respect to the initial V-notch - locally failed element, belonging to a propagating crack, and the nearest one within the given window along the front. It was shown, that this value scales with the change of the window's size as $z_{\max } \propto r^{\zeta}, \zeta$ being a 
roughness parameter. The possibility of the universal character of this parameter and its values in various cases are discussed in [21]. According to [19], experimentally obtained over a scale of several orders of magnitude (e.g., from $1 \mu \mathrm{m}$ to $1.5 \mathrm{~mm}$ ) values are: $\zeta=0.60 \pm 0.04$ for $8090 \mathrm{Al}-\mathrm{Li}$ alloy and $0.54 \pm 0.03$ for a Super $\alpha_{2} \mathrm{Ti}_{3} \mathrm{Al}$-based alloy. There could be possible a correlation between the region of roughness level and type of crack propagation [19].

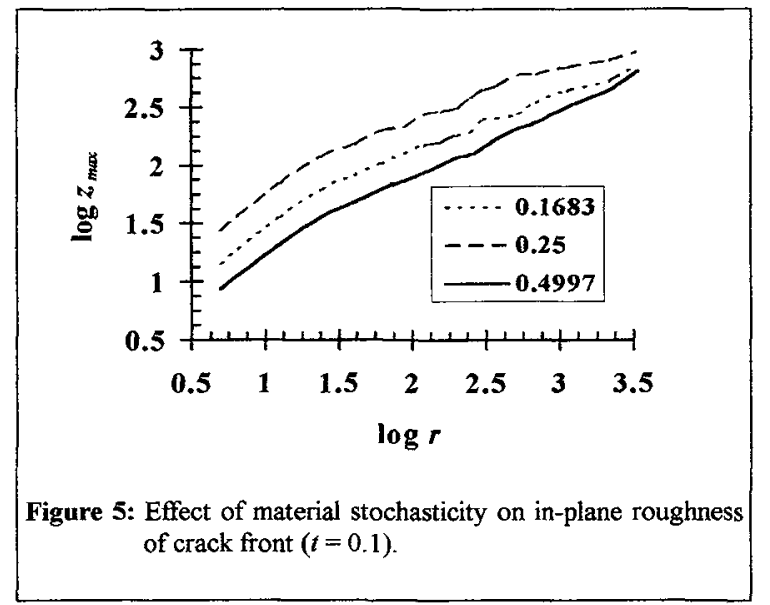

The treatment of data, characterizing the crack front morphology for three cases of stochasticity under study at various moments of time, has shown that the spatial randomness in material properties distribution effects also the roughness exponent:

\begin{tabular}{|c|c|}
\hline$K_{0.5}$ & $\zeta$ \\
\hline 0.1683 & $0.56 \pm 0.03$ \\
\hline 0.25 & $0.51 \pm 0.01$ \\
\hline 0.4997 & $0.62 \pm 0.01$ \\
\hline
\end{tabular}

The obtained values differ not sufficiently from some theoretical estimations, giving $\zeta=1 / 2$, but still the idea of universality of this parameter [22] should be additionally investigated.

\section{CONCLUSION}

Incorporation of local mechanisms into the model of crack propagation in brittle solids provides additional methods both for description and analysis of this complicated, highly non-uniform phenomenon. The approved self-similarity of such type of failure process can serve as a basement for elaboration of respective procedures and for introduction of additional parameters. Kinetics of damage evolution, randomly developing in a cross-section of the specimen with an initial notch, is responsible together with the crack-damage interaction - for the temporal features of crack propagation. Analysis of the effect of stochasticity in material properties' distribution on failure characteristics has shown that spatial scaling is directly related to a respective type of medium's randomness: more uniform materials have more ordered response (in terms of fractal dimension of crack front and stress distribution near cracks). In contrast, temporal scaling characteristics reflect another trend: a disorder smoothens - to a certain degree - processes of both crack growth and of energy release. Thus, more uniform media demonstrate higher levels of crack rate fluctuations and have wider spectra of singularities linked with more synchronized realization of local acts of elements' failure. And all three types of structures (specimens from Fig. 1) have the same scaling of the time-to-fracture - stress dependence, which has a multiple approval in experimental treatment of twin specimens. An in-plane roughness of crack fronts exhibit non-monotonous change with increase in material's spatial randomness.

\section{References}

[1] Hermann H.J. and Roux S. (Eds.), Statistical Models for the Fracture of Disordered Media (Elsevier Science B.V. North Holland, Amsterdam e.a., 1994).

[2] Liebowitz H. (Ed.), Fracture, Vol. I-VII (Academic Press, New York, 1968). 
[3] Knott J.F., Fundamentals in Fracture Mechanics (Butterworth, London, 1973).

[4] Kachanov M., "Elastic solids with many cracks and related problems". Advances in Applied Mechanics. J. W. Hutchinson and T.Y. Wu Eds. Vol. 30 (Academic Press, Boston e.a., 1994) pp. 259-241.

[5] Castaneda P.P., Willis J.R., J. Mech. Phys. Solids 43 (1995) 1919-1951.

[6] Silberschmidt V.V., Int. J. Fracture 56 (1992) R33-R38.

[7] Silberschmidt V.V., "Micro - macro transition for stochastic fracture process: fractal approach", MECAMAT 93. Int. seminar on micromechanics of materials. Moret-sur-Loing, 6-8 July, 1993 (Editions Eyrolles, Paris, 1993) pp. 72-82.

[8] Silberschmidt V.V. and Chaboche J.-L., Engng. Fracture Mech. 48 (1994) 379-387.

[9] Silberschmidt V.V., "Damage - crack interaction: constitutive model and fractal approach": Computational Plasticity. Fundamentals and Applications. D.R.J. Owen, E. Onate and E. Hinton Eds. (Pineridge Press, Swansea, 1992) pp. 1633-1643.

[10] Silberschmidt V.V., "Model of stochastic crack propagation - fractal and multifractal parameters of the process", Localized Damage III: Computer-Aided Assessment and Control. M.H. Aliabadi, A. Carpinteri, S. Kalisky and D.J. Cartwright Eds. (Computation Mechanics Publications, Southampton, 1994) pp. 545-552.

[11] Murakami Y. (Ed.), Stress Intensity Factors Handbook, 2 Vols. (Elsevier Science B.V. North Holland, Amsterdam e.a., 1987).

[12] Silberschmidt V.V., "Fractal and multifractal characteristics of stochastic brittle fracture", Fractals in the Natural and Applied Sciences, London, 7-9 September 1993, M.M. Novak Ed. (Elsevier Science B.V. North Holland, Amsterdam e.a., 1994) pp. 385-396.

[13] Silberschmidt V.V., Europhys. Lett. 23 (1993) 593-598.

[14] Silberschmidt V.V. and Yakubovich Yu.M., Int. J. Fracture 61 (1993) R35-R40.

[15] Halsey T.C., Jensen M.H., Kadanoff L.P., Procaccia I. and Shraiman B.I., Phys. Rev. A 33 (1986) 1141-1151.

[16] Arneodo A., Bacry E., Muzi J.F., Physica A 213 (1995) 232-275.

[17] Mach J., Mas F. and Sagués F., J. Phys. A.: Math. Gen. 28 (1995) 5607-5622.

[18] Yamaguti M. and Prado C.P.C., Phys. Lett. A 206 (1995) 318-322.

[19] Daguier P., Bouchaud E. and Lapasset G., Europhys. Lett 31 (1996) 367-372.

[20] Feder J., Fractals (Plenum Press, New York, 1988).

[21] Daguier P., Bouchaud E. and Lapasset G., paper in this volume.

[22] Bouchaud E., private communication. 